\title{
Quand le comportement découvrit les neurones...
}

L'institut de neurophysiologie et de psychophysiologie (INP) de Marseille (1963-1986)

\section{François Clarac et Jean Massion}

\section{(2) OpenEdition}

\section{Journals}

Édition électronique

URL : https://journals.openedition.org/histoire-cnrs/4963

DOI : 10.4000/histoire-cnrs.4963

ISSN : 1955-2408

Éditeur

CNRS Éditions

\section{Édition imprimée}

Date de publication : 31 décembre 2007

ISBN : 978-2-271-06560-5

ISSN : 1298-9800

\section{Référence électronique}

François Clarac et Jean Massion, « Quand le comportement découvrit les neurones... », La revue pour I'histoire du CNRS [En ligne], 19 | 2007, mis en ligne le 31 décembre 2009, consulté le 20 mai 2021. URL : http://journals.openedition.org/histoire-cnrs/4963; DOI : https://doi.org/10.4000/histoire-cnrs. 4963

Ce document a été généré automatiquement le 20 mai 2021.

Comité pour l'histoire du CNRS 


\title{
Quand le comportement découvrit les neurones...
}

L'institut de neurophysiologie et de psychophysiologie (INP) de Marseille (1963-1986)

\author{
François Clarac et Jean Massion
}

1 L'INP, créé officiellement le $1^{\text {er }}$ janvier 1963, fut initialement composé de sept départements, quatre de neurophysiologie et trois de psychophysiologie. Il en comprendrait dix, dix ans plus tard. Si les choix du doyen Georges Morin (1903-1979), élève d'Henri Hermann (1892-1972) de l'école de physiologie de Lyon, ont intéressé la neurophysiologie, Jacques Paillard (1920-2006) a voulu créer un institut de psychophysiologie comme l'avait conçu son maître Henri Piéron (1881-1964) afin de développer une nouvelle approche du psychisme qui permette à la psychologie de prendre place dans les sciences biologiques. Basée sur une approche pluridisciplinaire, la psychophysiologie devait prendre appui sur la physiologie, s'adresser à l'ensemble des comportements, humains mais aussi animaux, normaux et pathologiques. G. Morin sera le premier directeur de l'INP. J. Paillard, son adjoint, le deviendra en 1969. Les directeurs de départements, connus du doyen Morin, venaient pour certains de la faculté des sciences de Lyon, pour d'autres, du laboratoire que dirigeait à Paris, Pierre Paul Grassé (1895-1985), un des cofondateurs avec Henri Piéron (1881-1964) et Alfred Fessard (1900-1982) du certificat de psychophysiologie à la Sorbonne. Il n'y eut qu'un Marseillais, Robert Naquet (1921-2005).

L'organisation de l'INP

2 L'organisation de l'INP assez révolutionnaire pour l'époque, comprenait à coté des départements, un ensemble très performant de services techniques, communs, placés chacun sous la responsabilité d'un ingénieur ou d'un technicien (ITA). Le service d'animalerie et chirurgie dirigé par Pierre Lucciani (1935-1999) pouvait accueillir de nombreuses espèces animales dont des primates. Il disposait d'une salle d'opération moderne. On comptait aussi un secrétariat de direction, une bibliothèque, un service calcul, la microscopie électronique, le service d'histologie, celui de photographie, d'électronique, de radio-isotopes et de micromécanique. 
3 Le conseil de laboratoire, présidé par son directeur, était l'élément clé du fonctionnement de l'ensemble, se composant de représentants nommés et élus des départements et d'élus du collège des chercheurs et des ITA. Il délibérait sur la gestion de l'institut, la répartition des crédits, la gestion du personnel, et sur la politique scientifique. Au cours des conseils, J. Paillard faisait preuve d'une grande patience; les réunions pouvaient durer la journée entière ; on y voyait se révéler les caractères des uns et des autres et, en fin psychologue, J. Paillard, prenant un certain plaisir, concluait là où il le voulait !

Le département de psychophysiologie générale

4 Dirigé par J. Paillard, ce département constituait à lui seul un laboratoire pluridisciplinaire comprenant des psychologues, des psychophysiologistes, des neurophysiologistes, des ingénieurs, des physiciens, etc. Sa thématique scientifique consistait en l'étude spatio-temporelle des comportements moteurs et de leurs mécanismes neurophysiologiques, avec les concepts sous-jacents de programmation motrice, de rétroaction sensorielle, de l'organisation de l'espace et sa structuration par le mouvement actif. Ses travaux reposaient sur une réflexion théorique impliquant la notion d'« auto organisation » du vivant et en particulier du système nerveux ${ }^{1}$.

Dans ce cadre, les principales thématiques portaient sur la préparation à l'action, le déclenchement des programmes moteurs et le guidage de l'action, le rôle du mouvement actif dans le sens de la position et de la restructuration de l'espace visuo moteur, dans l'organisation de l'espace chez les jeunes aveugles, chez les handicapés moteurs et dans la modification des messages cutanés qui accompagnent le mouvement actif. Des chatons élevés en lumière stroboscopique ont permis l'analyse du rôle de la vision périphérique comparée à la vision centrale dans le développement du contrôle postural et dynamique. Les études réflexologiques s'appuyaient dans ce cadre général, sur le fuseau neuromusculaire dont J. Paillard faisait un modèle exceptionnel de régulation. Son premier élève marseillais a étudié chez le chat, le contrôle du cortex moteur sur la commande fusimotrice statique et dynamique. Il a même fait analyser la locomotion du crabe pour la présence chez ce crustacé, d'un récepteur comparable ce qui confirmait son intérêt pour les études comparées des comportements.

6 Issu de ce département, Jean Requin (1938- 1996) s'est vu confier en 1976 la direction du département de psychobiologie expérimentale. Il a su apporter dans l'analyse des processus de préparation à l'action des solutions originales, mettant en parallèle le problème de la localisation des fonctions cognitives et l'activité des ensembles cellulaires neuronaux. L'équipe a abordé aussi l'approche réflexologique de l'organisation $\mathrm{du}$ mouvement, l'estimation $\mathrm{du}$ temps, la coordination inter hémisphérique des activités bimanuelles et les processus attentionnels. En analysant l'activité unitaire corticale chez le Singe, J. Requin a construit un schéma d'organisation modulaire des processus qui conduisent de la préparation à l'action ${ }^{2}$.

Les départements de physiologie

7 Le département de neurophysiologie appliquée a été dirigé par R. Naquet. Élève d'Henri Gastaut (1915-1995), il a poursuivi sa formation chez Giuseppe Moruzzi (1910-1986) à Pise et chez Horace Magoun (1907-1994) à Long Beach. Il s'est ainsi familiarisé avec le système réticulé activateur et les phénomènes de veille, de sommeil et de conscience. Il a développé des études sur l'épilepsie et les drogues antiépileptiques et a mis en évidence, chez le chat, l'action anticonvulsivante des benzodiazépines, élément clé dans les médications antiépileptiques ${ }^{3}$. La découverte et l'étude de l'épilepsie 
photosensible du babouin (Papio papio) a été l'autre point fort de ses recherches. Il a lancé des recherches sur l'hyperbarie avec la Comex et a décrit le syndrome nerveux des hautes pressions. Sous-directeur de l'INP, il a quitté Marseille en 1973, pour prendre la direction de l'institut Alfred-Fessard à Gif-sur-Yvette.

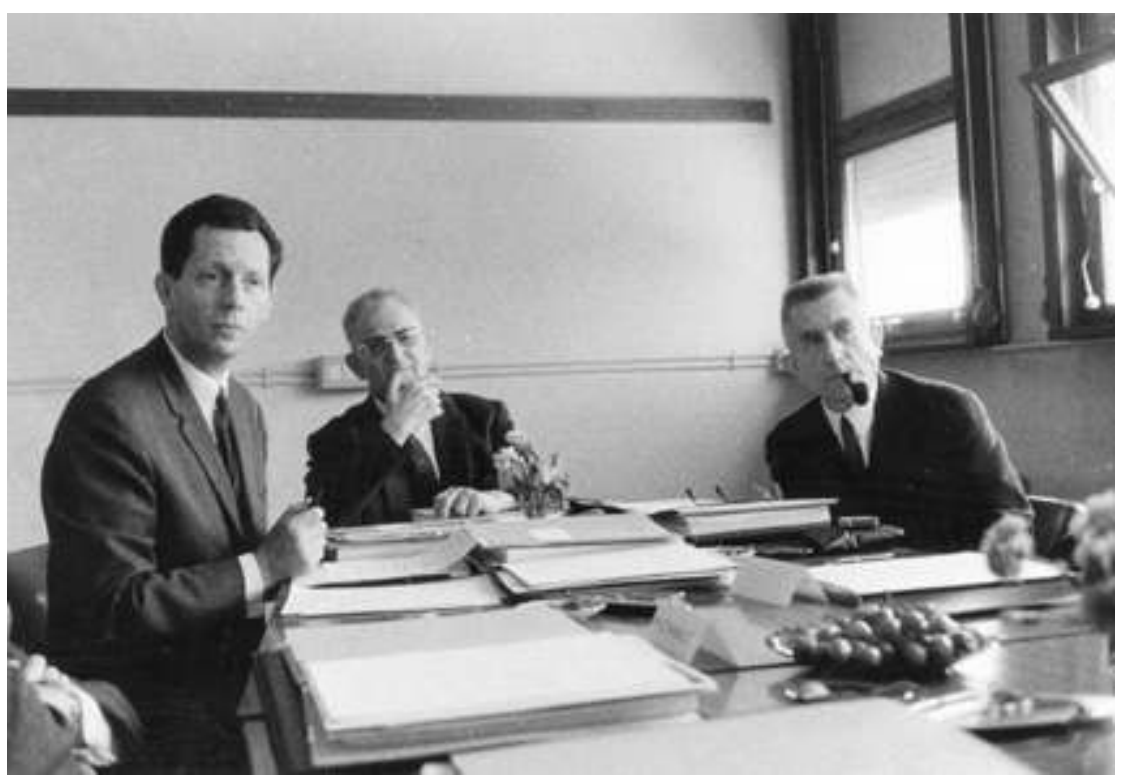

Premier conseil de l'institut en 1963. De gauche à droite : Jacques Paillard, Georges Morin et l'administrateur Dupuy. D.R.

8 Le département de neurophysiologie végétative, dirigé au départ par Michel Dussardier puis par Noël Méi, qui sera sous-directeur à partir de 1977, s'est intéressé à l'exploration des récepteurs vagaux et splanchniques, de leur projection centrale, de la motricité intestinale et de celle du cardia ${ }^{4}$. Il a favorisé les collaborations avec la faculté de médecine et la faculté d'odontologie et a ouvert la voie à un ensemble de recherches dans le domaine de la neurophysiologie végétative dont il fera un pôle important.

Le département de neurophysiologie générale a été confié au départ à Valentine Bonnet (1902- 1988). Après une thèse sur la rythmicité cellulaire chez Henri Cardot (1886-1942) à Lyon, elle a rejoint Frédéric Brémer (1892-1982) à Bruxelles, où ses travaux ont porté sur le cervelet, la réticulée et le cortex visuel. J. Massion, arrivé en 1967, a repris le département et a été sous-directeur de l'INP de 1973 à 1977. Il a lancé des recherches sur la physiologie des voies motrices (voies pyramidale, rubrospinale, cérébellothalamo-corticale et ganglions de la base) et sur la coordination entre posture et mouvement, l'apprentissage et la pathologie ${ }^{5}$.

Le département de neurophysiologie cellulaire a été dirigé par Angélique Arvanitaki (1901- 1983), puis par son mari, Nicolas Chalazonitis (1918-2004). Après une thèse également réalisée chez $\mathrm{H}$. Cardot, A. Arvanitaki a travaillé à la station maritime de Tamaris-sur-Mer où elle a collaboré avec A. Fessard. C'est là qu'elle a développé le modèle de l'aplysie, avec ses neurones géants, dont elle a identifié les activités intracellulaires. Après la disparition d'H. Cardot, elle a poursuivi ses travaux à la station océanographique de Monaco, obtenant une reconnaissance mondiale pour son modèle d'invertébrét. Les recherches du département étaient centrées sur l'électrogenèse des biomembranes.

11 Le département de neurobiologie comparée créé au $1^{\mathrm{er}}$ janvier 1976 avec l'arrivée à Marseille de Maurice Moulins (1936-1995) à la faculté des sciences de Saint Charles, a 
regroupé un ensemble de chercheurs intéressés par les activités sensori-motrices des invertébrés et leur substrat neuronique jusqu'à son départ pour Arcachon en $1978^{7}$. François Clarac, revenu à Marseille en 1988 a succédé à J. Paillard lors de son départ à la retraite. Il s'est consacré principalement à l'étude des bases neurobiologiques de la locomotion.

Les départements de comportement

Le département du comportement animal dirigé par Édouard Deleurance (1918-1990) comprenait deux thématiques: l'étude de la nidation des guêpes polistes avec une orientation éthologique et une orientation neuroendocrinienne, l'étude des coléoptères cavernicoles en recherchant les hormones régulant la mue de ces Insectes. Le rôle de l'épigenèse dans le contrôle du comportement de ponte a été précisé ${ }^{8}$, ainsi que l'approche endocrinienne du comportement du Grillon'.

De formation mathématique, Henri Durup (1930-2002) a analysé le comportement d'exploration du Hamster doré dans un labyrinthe à choix multiple. Il s'est vu confié en 1973 le département de psychologie animale avec pour thème d'étude l'exploration et l'orientation dans l'espace de lieux et sa modélisation chez les rongeurs.

14 Une telle approche a été développée aussi chez le Chien, le Chat et le Cheval. Une colonie de Babouins hébergée à Rousset a permis une étude de socio/éthologie chez le primate. Le département de psychophysiologie comparée avait une orientation éthologique affirmée. Il a été dirigé par Georges Le Masne, qui assurait, l'enseignement de psychophysiologie comparée à l'Université. Le département développait les thèmes suivants : le comportement social des Fourmis, le comportement sexuel des poissons amphibies périophthalmes, le comportement sexuel des isopodes terrestres et les rythmes d'activité locomotrice des chilopodes et diplopodes. Des travaux morphologiques et neurophysiologiques chez le comportement antennaire des fourmis, ont aussi été développés ${ }^{10}$.

Évolution de l'INP et quelques chiffres

L'évolution de l'INP s'est concrétisée par le dynamisme de la plupart des équipes et l'apport de nouvelles orientations. D'autres départements sont apparus comme celui d'André Calas et d'André Niéoullon. À la tête du département de neurobiologie cellulaire, de 1974 à 1981, A. Calas a introduit de nouvelles approches dans le domaine de la morphologie par l'utilisation de la radioautographie, de l'immunocytochimie et du traitement d'images sur le modèle du neurone à sérotonine. Il a décrit l'innervation sérotoninergique de la moelle, celle de l'aplysie, la bipotentialité de certains neurones du raphé (sérotonine, GABA). A. Niéoullon, travaillant sur la plasticité du système dopaminergique nigrostrié, occupera le poste universitaire de J. Paillard lors de sa retraite en 1989 et dirigera le département de neurochimie fonctionnelle créé après l'INP. Nicolas Franceschini, arrivé à l'INP en 1979, a développé des modèles adaptés de l'oeil composé de la mouche et de son système visuel. Ces études ont servi de point de départ à la réalisation de robots visuoguidés.

L'INP composé au départ de plus de 80 membres (41 chercheurs, 46 ingénieurs et ITA) en comprendra 159 en 1967 (93 chercheurs et 66 techniciens) et 184 la dixième année (104 et 80). Les dix années suivantes, environ 200 personnes seront présentes. En fait, un tel nombre ne montre pas le coté dynamique de cette population dont une grande partie n'était là que transitoirement. Parmi les chercheurs, plus d'une cinquantaine faisaient partie du CNRS. La production scientifique des vingt ans, a représenté pour les dix premières (1963-1972) : 20 doctorats d'État, 24 thèses de $3^{\text {e }}$ cycle et 226 articles 
scientifiques, et pour les dix suivantes (1973-1982) : 38 thèses d'État, 48 thèses de $3^{\mathrm{e}}$ cycle et 542 articles scientifiques. Des journées thématiques et des colloques internationaux ont assuré le rayonnement de l'institut.

17 La structure a du se réformer en 1986, en se divisant en deux laboratoires, l'un dirigé par N. Méi étudiant les mécanismes plus cellulaires (le laboratoire de neurobiologie, LNB), l'autre, composé d'équipes plus tournées vers la sensori-motricité et le comportement, le laboratoire de neurosciences fonctionnelles (LNF) dirigé par J. Massion.

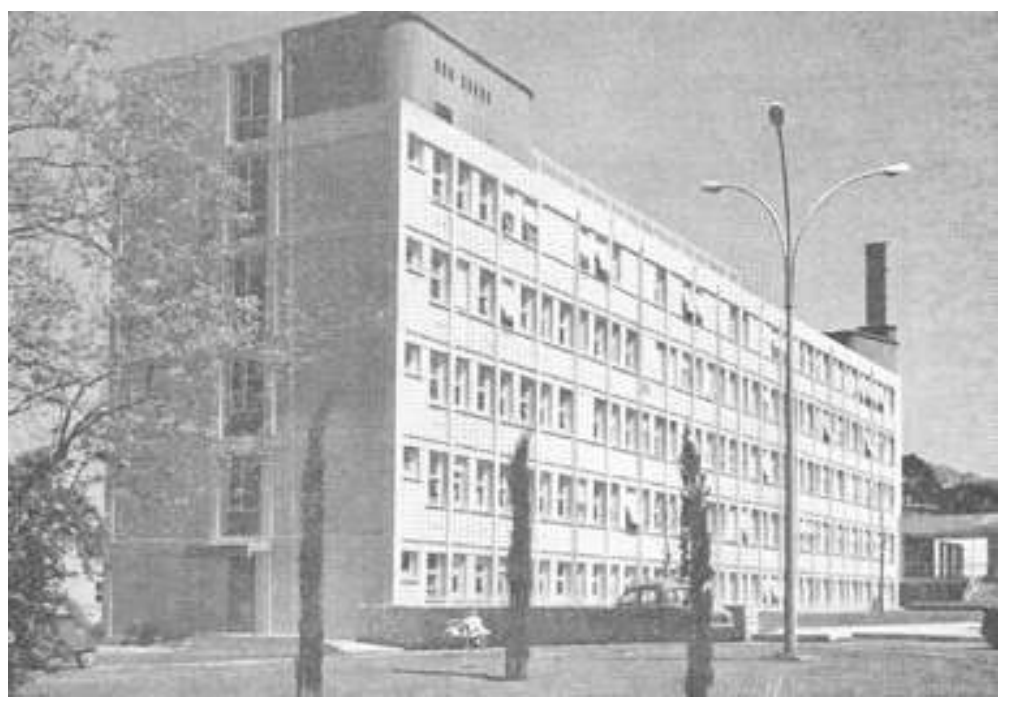

L'institut en 1963. D. R.

Le rayonnement national et international

L'évolution des concepts avait conduit au niveau européen à créer une nouvelle société, l'European Brain and Behavior Society, dont J. Paillard a été l'un des membres fondateurs. La première réunion a eu lieu à Marseille en 1969. L'INP, devenu lieu de passage pour les spécialistes des fonctions sensori-motrices, était à l'époque avec l'Institut für Hirnforschung de Zürich, dirigé par Konrad Akert, l'une des principales concentrations de scientifiques spécialisés dans ce domaine. Une telle réussite a permis l'organisation en 1973 à Aix-en-Provence d'un grand symposium international, intitulé «Comportement moteur et activités nerveuses programmées " ${ }^{11}$.

Les échanges internationaux ont été très nombreux à cette époque, aussi bien avec la plupart des pays d'Europe, qu'avec les États-Unis, le Japon et le Canada. L'INP comptait près de $15 \%$ d'étrangers parmi ses chercheurs. Il y eut des échanges privilégiés avec le Québec, initiés par Yves Lamarre et par Jean-Pierre Cordeau, directeur à l'époque du Centre de recherche neurologiques de l'université de Montréal. C'est d'ailleurs au Québec que J. Paillard effectuera l'essentiel de ses recherches après sa retraite. Les contacts avec les universités d'Oxford, l'école de Hans Kuypers (1925-1989) en Hollande, celles d'Anders Lundberg en Suède, de G. Moruzzi en Italie, de K. Akert en Suisse, ont concrétisé l'intérêt pour la motricité. Des programmes d'échanges ont été établis avec des laboratoires des pays de l'Est, comme l'école de Konorski, élève de Pavlov à Varsovie, comme les grands centres de recherche en Russie dérivés de l'école de Bernstein, et l'institut des activités nerveuses supérieures. C'est d'ailleurs dans des réunions bisannuelles organisées par l'école bulgare de physiologie à Sophia 
(Professeur Gantchev), que pouvaient se rencontrer les scientifiques occidentaux et orientaux évitant ainsi la coupure pesante du rideau de fer.

Conclusion

20 Le pari de J. Paillard de réaliser un institut pluridisciplinaire de niveau international, a été réussi. L'INP a bénéficié d'un environnement universitaire de $2^{\mathrm{e}}$ et de $3^{\mathrm{e}}$ cycles, de qualité. Il a, en retour, apporté un potentiel d'accueil et de formation essentiel aux structures universitaires et a favorisé l'éclosion de nouvelles formations, comme celle de la faculté des sciences du sport à Luminy ${ }^{12}$. Au total, alors que le concept de neurosciences émergeait à peine, l'INP a été un acteur puissant dans l'évolution de la pensée scientifique française et internationale dans les années 1960-1980.

Deux figures se détachaient à la faculté de médecine en neurologie, Henri Gastaut (1915-1995), célèbre dans le monde entier pour ses travaux en électroencéphalographie, et le professeur Georges Serratrice qui analysait les maladies dégénératives neuromusculaires. À la faculté des sciences de SaintCharles, le professeur Paul Benoît, qui avait créé un laboratoire de physiologie sur les fibres nerveuses et musculaires, a favorisé, en 1957, l'installation de J. Paillard, nommé professeur de psychophysiologie et en 1960, celle de M. Dussardier, professeur de neurophysiologie. Ces nouveaux laboratoires universitaires s'installeront peu de temps après, au nord de Marseille sur le campus de SaintJérôme. J. Paillard, remplacé par Maurice Hugon, quittera ses fonctions en 1967 pour devenir professeur à Luminy.

\section{NOTES}

1. Paillard J. «Système nerveux et fonction d'organisation ». In Psychologie, Piaget J., Mounoud P., Bronckart J.-P., eds. Encyclopédie de la Pléiade, Gallimard, 1986, pp. 1378-1441.

2. Requin J., Rielhe A., Seal J. Neuronal activity and information processing in motor control: from stages to continuous flow. Biol. Psychol., 1988, 26(1-3), pp. 179-98.

3. Lanoir J., Plas R., Naquet R. Étude de neurophysiologie comparative de trois substances psychotiques. J. of Physiol., 1963, 55, pp. 281-282.

4. Mei N. Mécanorécepteurs vagaux digestifs chez le chat. Exp. Br. Res., 1970, 11, pp. 502-514.

5. Massion J. The mammalian red nucleus. Physiol. Rev., 1967, 47(3), pp. 383-436.

6. Arvanitaki A., Chalzonitis N. "Excitatory and inhibitory processes initiated by light and infra-red radiation in single nerve cells". In Nervous inhibition, Florey E. ed. Pergamon Press, 1961, Oxford, London, pp. 194-231.

7. Clarac F., Moulins M., Vedel J.-P. Rhythmical motor activity; central and peripheral neurophysiological mechanisms. J. Physiol., 1977, 73, n4, pp. 405-616.

8. Gervet J. Oviposition et sa régulation dans la société polygénique de polistes gallicus. Behaviour, 1965, 25 (3), pp. 221-233.

9. Strambi A., Strambi C. Étude histochimique et ultrastructurale de la sécrétion de péricaryons neurosécrétoires de la pars intercerebralis chez la guêpe poliste. Acta Histochemica, 1973, 46 (1), pp. 101-109. 
10. Masson C. Mise en évidence au cours de l'ontogénèse d'une fourmi primitive (mesoponera caffraria), d'une prolifération tardive au niveau des cellules globuleuses ('globuli cells') des corps pédonculés. Z. Zellforsch, 1970, 106, pp. 220-231.

11. Paillard J., Massion J. Motor aspects of Behaviour. Comportement moteur et activités nerveuses programmées. Brain Research special issue, Elsevier, Amsterdam, 1974, 71, № 2, 3. 12. Niéoullon A. « Marseille et les neurosciences ». In Vingt six siècles de médecine à Marseille. Serratrice G. éd. Jeanne Lafitte, Marseille, 1996, pp. 707-715.

\section{RÉSUMÉS}

Dans les années 1960, Pierre Drach, alors directeur adjoint du CNRS pour les sciences exactes, préconise, dans le cadre du 3e plan, la création d'un institut de psychophysiologie. François Clarac et Jean Massion reviennent sur la genèse d'un institut qui profitait de la décentralisation et de la présence d'un pôle fort en neurologie à Marseille.

François Clarac and Jean Massion recount the creation of an institute dedicated to psychophysiology and neurophysiology in the 1960s in Marseille.

\section{AUTEURS}

\section{FRANÇOIS CLARAC}

François Clarac est directeur de recherche émérite, membre de l'unité «Plasticité et physio-pathologie de la motricité »(P3M).

\section{JEAN MASSION}

Jean Massion est directeur de recherche émérite au CNRS. 